\title{
Presentación del proyecto de normas transnacionales del proceso civil(")
}

\section{Antonio Gidi}

\section{El proyecto}

La Revista «DERECHO-PUC» ha tenido a bien publicar en este número las «Normas Transnacionales del Proceso Civil», proyecto cuya autoría corresponde a los Profesores Geoffrey C. Hazard Jr. y Michele Taruffo.

Sea esta la ocasión para presentar al profesor Taruffo a los lectores latinoamericanos. Sus enseñanzas ya están definitivamente incorporadas a nuestra ciencia procesal, sobre todo a través de sus obras y diversos artículos sobre el Derecho Comparado, la Motivación de la Sentencia, los Recursos y la Teoría de la Prueba. Recientemente ha publicado, en coautoría, un texto sobre el curso de Derecho Procesal Civil con características innovadoras. Es profesor titular en la Facultad de Derecho de la Universidad de Pavia, Italia, donde enseña los cursos de Instituciones del Derecho Procesal Civil y Derecho Procesal del Trabajo, y dirige al mismo tiempo el Instituto de Derecho Procesal Civil Comparado. Fue también Profesor Visitante en la Universidad de Cornell, en los Estados Unidos.

Geoffrey Hazard Jr. es profesor de Derecho Procesal Civil y de Ética Profesional en la Facultad de Derecho de la Universidad de

(*) Adaptado de la intervención realizada en el Coloquio Internacional "Processi di integrazioni e soluzione delle controversie: dat contenzioso fra gli stati alla tutela dei singoli: Europa e América Latinas, realizado en la Universidad de Roma Tor Vergata por invitación del Prof. Bruno Sasani. El autor desearía agradecer a sus amigos, Prof. Francesco Luiso, por la corrección del texto original al italiano, y Prof. Aníbal Quiroga León, por la amable invitación a publicar nuestro trabajo en esta prestigiosa revista.

** Traducción libre del Dr. Aníbal Quiroga León, Editor General de la Revista DERECHO-PUC. 
Pennsylvania (Filadelfia), y lo fue por muchos años en las universidades de Yale (New Haven) y de California (Berkeley). Es Director Emérito del American Law Institute (ALI). Ha publicado numerosos libros y artículos sobre el Derecho Procesal Civil y sobre la Ética Profesional, siendo en la actualidad uno de los más respetados juristas norteamericanos. Posee al mismo tiempo una gran experiencia práctica, académica y legislativa, habiendo sido el principal responsable (Relator) de la redacción del Restatement (second) of Judgments ${ }^{1}$ del American Law Institute, y de las Model Rules of Professional Conduct ${ }^{2}$ del American Bar Association. Es Miembro de la Comisión designada por la Suprema Corte Federal encargada de la Propuesta de Reforma y Perfeccionamiento del Proceso Civil en los Estados Unidos (Advisory Committee on Civil Rules).

Podemos decir, entonces, que este trabajo es en realidad una continuación de una obra que los autores escribieron en conjunto en 1993 sobre el Proceso Civil norteamericano. ${ }^{3}$

El proyecto es patrocinado por el American Law Institute (ALI). Es el ALI una asociación, fundada en 1923 , y compuesta aproximadamente por 3,400 miembros, de los cuales la mitad son abogados en ejercicio, y los demás jueces y profesores en disciplinas jurídicas. Se trata de una entidad única en el mundo, por su marcada participación en la reforma, desarrollo y perfeccionamiento del derecho norteamericano. Entre las obras más importantes que han sido producidas por este Instituto están innumerables Restatements ${ }^{4}$, o el Uniform Code y el Model Penal Code. 5

1 Segunda edición de la Compilación (análisis y comentario) de Jurisprudencia norteamericana realizada por el American Law Institute. N. del Traductor.

2 Normas Modelo del Comportamiento Profesional; N. del Traductor.

3 Cf. American Civil Procedure. An Introduction, Editorial de la Universidad de Yale, 1993. El libro fue publicado en el Japón, en traducción a cargo del Prof. Makoto Tanabe.

V. la reseña del Prof. Antonio Gidi, publicada en Génesis. Revista de Direito Processual Civil., Vol. 6, p. 851-5, esp. p. 852.

4 "Trabajos de doctrina, elaborados por el American Law Institute, que exponen y analizanen forma similar a un códifo comentado, las principales reglas jurídicas de origen jurisprudencial aplicables en los Estados Unidos en distintas materias" Guillermo Cabanellas: Lexis (English-spanish) legal dictionary; Butterworth Legal Publishers; 1991, T. I; p. 538 (N. del Traductor).

5 Sobre la importancia del «American Law Institute» en los Estados Unidos, con especial énfasis en los Restatements, cf. Ugo Mattei, II modello di common Law, pp. 161-3. Ver también: Geoffrey Hazard Jr., The American Law Institute: what it is and what it does Centri di Studi e Ricerche di Diritto Comparato e Straniero (CNR), 1994. 
$\mathrm{Al}$ inicio de 1999, el proyecto pasó a ser conducido en colaboración con UNIDROIT (The International Institute for the Unification of Private Law). Como se sabe, el UNIDROIT es una organización intergubernamenal independiente, fundada hace más de 70 años, y cuyo objetivo es armonizar y unificar el Derecho Privado a través de tratados y convenciones internacionales y leyes-modelo. El UNIDROIT está compuesto por casi 60 países-miembros y tiene su sede en Roma.

\section{Objetivos}

No es preciso comprobar aquí el hecho notorio de que las transacciones internacionales se han multiplicado, y que también el derecho procesal necesita acompañar la evolución de la sociedad. Se dice que el mundo se está encogiendo. Se habla de la globalización y de la libre circulación de las mercaderías y el comercio. Con menos frecuencia, se habla también de la libre circulación de las personas y los trabajadores. En todo caso, las fronteras físicas que existen entre los países no tienen más el mismo significado que hace 15 ó 10 años atrás, y que los litigios de dimensión internacional son cada vez más frecuentes en los tribunales de las grandes capitales.

La necesidad de una uniformización de las normas procesales se hace sentir ya desde algún tiempo, sobre todo para el abogado habituado a operar en causas de naturaleza transnacional. Esa necesidad está acentuada por el hecho de que, por el momento, las tentativas de armonización del derecho se han limitado casi exclusivamente a materias de contenido sustantivo. Las pocas convenciones internacionales en el área del derecho procesal se han limitado a estimular la asistencia jurídica entre los países, principalmente en lo que se refiere a las notificaciones y a la actuación probatoria en otro país, así como al reconocimiento y ejecución de sentencias extranjeras ${ }^{6}$. El procedimiento propiamente dicho continúa siendo reglado por el derecho procesal interno de cada país.

6 Cf., por ejemplo, The Hague Convention of Taking of Evidence Abroad (Convención de la Haya sobre la obtención de pruebas en el extranjero") y también la aún no acabada International Jurisdiction and Foreign Judgments in Civil and Commercial Matters (Jurisdicción Internacional y Sentencias Extranjeras en Asuntos Civiles y Comerciales). Sobre el motivo de esta indiferencia de la ley procesal, ver las opiniones de Catherine Kessedjian, First Impression 
La normas transnacionales vienen a uniformizar $o$ armonizar las normas procesales actualmente existentes en los diversos países, en lo que se refiere a las controversias transnacionales. Es decir, su principal objetivo consiste en reducir la falta de certeza e inseguridad del extranjero que se ve obligado a litigar en un foro judicial que no le es familiar, a través de leyes procesales que desconoce. Su inserción en los sistemas jurídicos de los diversos estados puede ser hecha tanto a través de un tratado internacional, como a través de la promulgación de una ley nacional.

En la práctica, se trata apenas de la creación de uno más, de los numerosos «procedimientos especiales» comunes en todos los sistemas procesales. Sucede que, en este caso, el proceso no es especial en razón de la especificidad de la situación del derecho material objeto del proceso, sino tan solo en base al carácter transnacional de la controversia, sea cualquiera la naturaleza que tenga. Así, es importante observar que, dentro del ámbito previsto en el Art. $1^{\circ}$ (a), de las normas tienen un carácter "transubstancial", siendo por tanto aplicables en cualquier tipo de controversias.

Las normas transnacionales son acompañadas de un comentario offcial. Todavía, por limitaciones de espacio, los comentario serán reproducidos en esta oportunidad. Aquellos interesados podrán consultar su texto original en inglés, acompañado de una introducción y comentario artículo por artículo, en la Página Web del American Law Institutę

Como se sabe, la uniformización del derecho procesal civil no es una idea pionera. En los últimos años han florecido bien intencionadas propuestas de uniformización del proceso civil en América Latina y en la Comunidad Europea ${ }^{8}$.

Las normas transnacionales son una propuesta a un solo tiempo, más amplia y menos ambiciosa que el Código Procesal Civil Modelo para Iberoamérica y la Aproximación a la Ley judicial en la Comunidad Europea. Se trata de una propuesta más amplia, en la medida que su aplicación no está circunscrita a países situados en una única región y ligados por una historia y culturas comunes. Por ello, al mismo tiem-

of the Transnational Rules of Civil Procedure from Paris an The Hague, 33 Text. Int'l L.J. 477-80 (1998) y de Geoffrey C. Hazard Jr.,From whom no secrets are hid, 76 Texas L. Rv. 1665,1667 (1998).

7 CF. http://www.ali.org/ali/transrules.htm

8 CF. "Anteproyecto del Código Procesal Civil Modelo para Ibero América», Revista del Proceso, Vols. 52 y 53, 1988 y 1989, y Marcel Storme (Ed.), Aproximation of judiciary law in the European Union, Kluver, 1994. 
po, se trata de una tarea menos ambiciosa, en la medida que su focus está limitado solo a los litigios considerados "transnacionales", no alcanzando a los litigios domésticos, que continuaran regidos exclusivamente por la ley procesal nacional.

Además, se vislumbra que el proyecto puede ser utilizado como base para el procedimiento a ser adoptado en los arbitrajes internacionales. Como se sabe, las actuales normas sobre arbitraje guardan silencio en lo que se refiere al procedimiento. Así, en la práctica, las reglas procesales de un arbitraje dependen, en gran medida, de la nacionalidad del árbitro que presidirá el tribunal arbitral, que naturalmente optará por el sistema por el cual estuviera más familiarizado. Así, un arbitro inglés adoptara las Rules of Court inglesas, y un Alemán usará el ZPO como estructura procedimental de base para la conducción del arbitraje y para sus decisiones de contenido procesal. La adopción de las normas transnacionales podrá traer una cierta uniformización de los procedimientos de arbitrajes transnacionales. Las partes contratantes podrán libremente adoptar las normas transnacionales como aquel procedimiento a ser adoptado en su arbitraje, independientemente de quién o cuál será la persona u organización encargada de arbitrar esta controversia.

Una cuestión más delicada, y que está en la consideración de lo relatores en este momento, es definir aquello que debe ser considerado como litigio «transnacional» y como éste debe ser objeto de definición en el proyecto. La posición actualmente adoptada por las normas es la de considerar como "residencia habitual" de las partes aquella en donde está localizado el bien objeto del litigio. Esta es todavía una cuestión polémica que, sin duda, podrá ser modificada en las posteriores versiones?.

Es imperativo observar que las normas transnacionales no son propiamente un código, en el sentido que el derecho continental europeo da a tal expresión: Se trata tan solo de un grupo sistemático de normas procesales. El objetivo de los relatores es el de seleccionar algunos de los problemas más relevantes en la conducción de la controversias transnacionales y otorgarles una disciplina jurídica uniforme, sólo para aquello que pueda ser considerado esencial en los litigios transnacionales. Las cuestiones no reguladas por las normas son dejadas a la solución

9 Cf. Normas Transnacionales, Art. 1(a). Igualmente está en fase de discusión si las Normas Transnacionales deben aplicarse únicamente a las controversias de naturaleza comercial. Hay una tendencia en este momento a extender su ámbito de aplicación a cualquier controversia de naturaleza civil, financiera o comercial. 
de la ley local. ${ }^{10}$ En eso viene a nuestro encuentro las enseñanzas del Prof. Konstantinos Kerameus, según las cuales la unificación internacional del derecho procesal solamente podrá ser parcial. ${ }^{11}$

Un aspecto que amenaza la legitimidad del proyecto es que en un importante número de casos las normas transnacionales no podrán ser adoptadas plenamente en los Estados Unidos. En primer lugar, sobre la base de que es una garantía constitucional la presencia del jurado en algunas controversias de naturaleza civil. ${ }^{12}$ El tribunal por jurado exige un proceso hasta cierto punto incompatible con la uniformización propuesta por el proyecto. La existencia misma del jurado puede ser considerada como un drástico abandono del ideal de una uniformización pretendido por las normas del proyecto.

Además, las normas necesitan ser reconocidas y adoptadas por todos los estados de los Estados Unidos, so pena de regir solo apenas en la esfera federal. Como sabemos, sobre la base de la peculiaridad del sistema federal americano, cada uno de sus cincuenta estados dispone, no solamente de un poder judicial autónomo, sino también de una legislación procesal diferente. Así, los tribunales federales aplican una misma ley procesal federal y los tribunales estatales aplican sus leyes procesales en vigencia en el estado de que se trate. ${ }^{13}$

Estos riesgos están potencializados por la circunstancia de que, en la mayoría de las causas regladas por las normas transnacionales, cualquier

10 Cf. Normas Transnacionales, Art (6)

11 Cf. Konstantinos Kerameus, Procedural Unification, the need ant the limitations en I.R. Scott (ed.), International perspectives, on civil justice, p. 54, 1990. Un reflejo de esta concepción y de la realidad del proyecto de armonización fue realizado por la Comisión Storme. Cf. Marcel Storme (ed.), Approximation of judiciary law in the European Union, cit. Naturalmente, se trata de la situación actual, pero ciertamente ello tendrá cada vez menos relevancia. Creemos, que en un futuro próximo el error de la afirmación antes citado quedará de manifiesto.

12 El derecho a un juicio por jurado en las controversias de naturaleza civil está previsto en la Sétima enmienda de la Constitución Norteamericana y su actual concepción ha sido desarrollada en importantes decisiones de la Suprema Corte Federal. En general, la competencia en un «juicio por jurado" se limitará únicamente a aquellas controversias donde la pretensión del actor fuera de naturaleza indemnizatoria, no así en aquellos casos donde el actor solicite una medida cautelar (orden de innovar o de no innovar). Este es un tema complejo que nos lleva a la histórica distinción entre los ya extintos sistemas de la Equidad y el Derecho. Cf. Antonio Gidi, reseña, Génesis. Revista de Direito Processual Civil. Vol. 6, p. 853.

13 Op. Cit. p. 855 
parte tiene derecho a invocar el tribunal por jurado y la mayoría de estas controversias son competencia de la justicia estatal ${ }^{14}$.

Este efecto está potencializado, además, por el hecho de que las normas otorgan al juez de primer grado una flexibilidad y discrecionalidad que como juez del Civil Law no está habituado a operar, así, se corre el riesgo de generar aún más discrepancias, incertidumbres y desorden de aquello que preexiste al proyecto. Ese problema solamente podrá ser aliviado a través de la implementación de un tribunal superior único, que tenga como objetivo la uniformización de la interpretación. ${ }^{15}$

Será extremadamente difícil justificar en el presente momento histórico, la creación de un tribunal supranacional para la resolución de controversias exclusivamente privadas. Sin embargo, la creación de un tribunal central de apelación en cada país, especializado en causas internacionales, puede servir de instrumento uniformizador, principalmente si los diversos tribunales nacionales tuvieran conocimiento de las decisiones emitidas por los tribunales de otros países y las usasen como precedentes, aún cuando sin efecto vinculante.

\section{Histórico}

Felizmente las normas transnacionales habían sido concebidas algunos años atrás, ${ }^{16}$ desde el inicio de 1998, cuando comenzó el patrocinio del American Law Institute, y el desarrollo del autor del proyecto, cuyo texto ya fue discutido oficialmente en Bologna, Philadelphia Vancouver, San Francisco, Tokio y Singapur. ${ }^{17}$ Participaron en ese evento, abogados especializados en litigios internacionales, jueces y profesores del derecho procesal civil y derecho internacional privado de diversos países.

14 Será competente la Justicia Federal solamente en el caso de una completa diversidad de ciudadania. Ese es un tema particular de la organización constitucional de la justicia Norteamericana, que no podrá ser tratado en esta oportunidad. Cf. James, Hazard Jr. y Leubsdorf, Civil Procedure, p. 97.

15 Cf. Konstantinos Kerameus, "Procedural Unification: The need and the limitations", en International perspectives, on civil justice; I.R. Scott (Ed.) p. 54, 1990.

16 Antes de su desarrollo en ALI, el Proyecto ya había sido presentado por Hazard y Taruffo en Tilbur, Bologna, Florencia, Paris, Cornell y Philadelphia. Cf. Michele Taruffo, Drafting rules for transnational litigation, ZZPint'l L.J. 449 (1997).

17 El Encuentro en Beijing fue suspendido a causa del bombardeo de la embajada China en Belgrado por la NATO. 
Entre los consejeros (advisers) están los nombres más representativos en el área del proceso civil en el mundo tales como: Bryan Beaumont, Stephen Burbank, Federico Carpi, Edward Cooper, Peter Gilles, Stephen Goldstein, J. A. Jolowicz, Mary Kay Kane, Benjamín Kaplan, Catherine Kessedjian, Takeshi Kojima, Andreas Lowenfeld, Ramón MulleratBalmaña, Yasuhei Taniguchi, Gerhard Walter, Garry Watson y Charles Alan Wright, para citar apenas los más conocidos entre nosotros.

Los encuentros involucran a un grupo muy limitado de personas, por lo general reunidos en torno a una mesa grande. Este número reducido es cuidadosamente seleccionado y estimula a los participantes a intercambiar informaciones de alto nivel, creando una atmósfera de informalidad inexistente en los grandes congresos. En todos los encuentros ya realizados, los participantes contribuyeron con críticas y sugerencias muy importantes para el actual contenido de las normas transnacionales. Una simple comparación entre la versión original y la actual revela una evolución que solamente puede ser explicada por haber incorporado las críticas y sugerencias hechas por los participantes en los diversos encuentros, al punto de que el producto final puede ser considerado como una obra sustancialmente diversa de la que fue iniciada.

La primera versión oficial de las normas transnacionales como un proyecto del American Law Institute, se publicó en marzo de 1998 (primer borrador preliminar) y fue discutida en Bologna y Philadelphia. Las sugerencias y las críticas efectuadas por los participantes fueron tan numerosas que el $1^{\circ}$ de agosto fue necesario imprimir una nueva versión, especialmente para ser presentada en el encuentro de la Intenational Bar Association, realizado en Vancouver (Interim Revision). ${ }^{18}$

En el encuentro de Vancouver, recibimos aún más críticas y sugerencias y otros tantos cambios fueron introducidos en el proyecto. También recibimos sugerencias por escrito, lo que facilitó el trabajo de identificar los problemas y acoger las sugerencias. Una vez más, una nueva versión mejorada fue producida para los debates realizados en San Francisco Tokio, Singapur y Viena. Esta versión se tituló Discusion Draft, fue publicada el primero de abril de 1999 y es la que ahora se presenta al

18 Las versiones anteriores del Proyecto fueron publicadas en 30 Cornell Int'll L.J. 89 (1997), con una introducción del Prof. John Barcello III; y, en 33 Tex. Intll L.J. 499 (1998), un volumen especial, con una colección de artículos sobre las Normas Transnacionales. 
lector peruano. Otras versiones, aún más perfeccionadas, serán publicadas en el futuro inmediato, siempre con el objetivo de estimular su pública discusión, recibir nuevas críticas y mejorar aún más el proyecto.

El 24 de agosto de 1999, el Prof. Hazard fue relator general del XI Congreso Mundial de Derecho Procesal, realizado por la Asociación Internacional de Derecho Procesal, en Viena, y en septiembre del mismo año, presentó el proyecto en el Congreso de la International Bar Asociation que se llevó a cabo en Barcelona.

Futuros encuentros están siendo planificados en breve para América Latina, Asia, Europa y los Estados Unidos. Antes de que el proyecto sea cerrado, probablemente entre los años 2002 y 2003, se llevarán a cabo muchos otros encuentros. Como se ve, se trata de un proyecto que debe ser concluido en el mediano plazo. Sus efectos en la práctica del litigio transnacional, por otra parte, sólo podrán ser apreciadas dentro de muchos años.

El proyecto está en pleno camino y la participación de los procesalistas de América Latina es aún incipiente. Se espera que la iniciativa de la Revista de DERECHO PUC, de publicar el texto del proyecto en el Perú, con una traducción al español, sea un estímulo para una mayor participación de los procesalistas peruanos en este importante proyecto. ${ }^{19}$

Con el objetivo de estimular la amplia divulgación del proyecto y su discusión internacional, fueron preparadas diversas traducciones de las normas transnacionales. Así, Gerald Walter, de la Universidad de Berna, ha efectuado una traducción para el alemán; Koichi Miki, de la Universidad de Keio (Tokio) lo ha hecho para el japonés; Francesca Cuoma y Valentina Riva de la Universidad de Estudios de Pavia, para el italiano; Evaluz Cotto, de la Escuela de Derecho de la Universidad de Puerto Rico, Francisco Málaga, de la Universidad Pompeu Fabra (Barcelona), Anibal Quiroga León, de la Pontificia Universidad Católica del Perú (Lima), Horacio Segundo Pinto de la Universidad Católica Argentina, para el español; y Antonio Gidi de la Universidad de Pennsylvania para el portugués. Traducciones en otros idiomas están también en camino y en breve serán publicadas. Como se puede ver, hasta el momento disponemos de varias traducciones para el español, traducciones ligeramen-

19 Se invita a los interesados a efectuar una atenta lectura del Proyecto y a enviar sus críticas, sugerencias o comentarios en inglés o en español al American Law Institute, cuya dirección esta en nota de pie de página de la traducción. 
te diferentes serán publicadas en España, Puerto Rico, en el Perú, en Argentina y en otros países de América Latina. Al final del proyecto, sin embargo, habrá una única traducción española oficial que se realice a través del consenso de los diversos traductores.

Como se puede ver, no se trata de una tarea efectuada en apenas cuatro años. La activa participación de numerosos juristas, sea a través del debate oral, sea a través de la presentación de sugerencias escritas, minimiza la importancia de la participación exclusiva de cualquier persona y hace de este proyecto una tarea colectiva, dentro de los moldes de los otros proyectos emprendidos por el American Law Institute.

El debate de la doctrina sobre las normas transnacionales ya comenzó a florecer e importantes artículos sobre el tema ya fueron escritos. ${ }^{20}$ Las opiniones no siempre han sido favorables. Siempre que fue posible, sin embargo, las críticas han sido enfrentadas por los relatores y el proyecto ha evolucionado considerablemente, principalmente en función de tales comentarios. Todo lo cual indica que el proyecto promete continuar evolucionando hasta la publicación de su versión final.

20 Cf. Cf. Gary Born, Critical observations on the draft Transnational Rules of Civil Procedure, 33 Tex. Int'll L.J. 387 (1998), Russell J. Weintraub, Critique of the Hazard-Taruffo Transnational Rules of Civil Procedure, 33 Tex. Int'll L.J. 413 (1998), Jacob Dolinger and Carmen Tiburcio, The forum law rule in international litigation - which procedural law governs proceedings to be performed in foreign jurisdictions: lex fori or lex diligentiae?, 33 Tex. Int'll L.J. 425 (1998), Gerhard Walter and Samuel P. Baumgartner, Utility and feasibility of Transnational Rules of Civil Procedure: some German and Swiss reactions to the Hazard-Taruffo Project, 33 Tex. Int'll L.J. 463 (1998), Catherine Kessedjian, First impression of the Transnational Rules of Civil Procedure from Paris and The Hague, 33 Tex. Int'll L.J. 477 (1998), 33 Tex. Int'l L.J. 477 (1998), Geoffrey C. Hazard, Jr., Transnational Rules of Civil Procedure: Preliminary Draft No. 1, 33 Tex. Int'l L.J. 499 (1998), Michele Taruffo, Drafting rules for transnational litigation, ZZPInt' L.J. 449 (1997), Joaquim J. Forner Delaygua El proyecto del Instituto Americano de Derecho "Código Internacional Procesal Civil» y la cooperación judicial, inédito, 15 p. Y Ramón Mullerat y Joaquin J. Forner Delaygua, "Transnational Rules of Civil Procedure; una iniciativa del American Law Institut», ${ }_{2}$ en: Revista española de Derecho internacional, vol. 50, p. 376, 1998.

Ver también: Ricachard Marcus, Retooling American discovery for the twenty-first century: toward a new world order?, en: 7 Tulane Journal of International and Comparative Law p. 153 (1999), "Discovery and the role of the judge in civil law jurisdictions" en: 73 Notre Dame L. Rev, idem, "From whom no secrets are hid", en: 76 Texas L. Rev. p. 1665 (1998) e idem, Civil litigation without frontiers: harmonization and unification of procedural law, $X I$ World. Congress on Procedural Law, p. 1. 


\section{IV.Problemas}

Para comprender mejor el proyecto, es necesario analizar cuidadosamente los obstáculos que éste debe superar, antes de poder ser considerado como una alternativa viable a la realidad actual. Ese análisis es de fundamental importancia, principalmente en base a algunas críticas que el proyecto viene recibiendo, muchas de ellas sustancialmente desenfocadas, por la falta de una rigurosa visión crítica sobre los problemas envueltos.

En primer lugar, hay que evaluar la necesidad de crear un sistema procesal armónico para la resolución de las controversias transnacionales. Se trata de una decisión predominantemente política y que debe ser analizada objetivamente, en función de la realidad práctica actual.

En segundo lugar, es necesario evaluar la posibilidad de crear un sistema procesal armónico que sea útil y aceptable en países de diferentes culturas. Se trata de una cuestión de naturaleza predominantemente técnico-jurídico.

Superadas estas dos cuestiones es preciso aún evaluar el mérito de este proyecto en la creación de un sistema procesal que sea útil, armónico y aceptable. Sobre este enfoque se debe evaluar no solamente las soluciones dadas por las normas sino principalmente los problemas relacionados para el tratamiento uniforme. También se trata de un problema predominantemente técnico-jurídico.

Aun si todas estas cuestiones antes referidas fuesen respondidas afirmativamente, esto es, aun cuando un sistema armónico sea necesario y posible y aun cuando este proyecto este apto para crear un sistema que sea útil y aceptable en países de culturas diversas, hay una cuestión importante que requiere ser previamente resuelta. Se trata de su conveniencia, una cuestión de naturaleza exclusivamente política y que interesa más de cerca a los países subdesarrollados y en vías de desarrollo como, por ejemplo, los países de América Latina. Esta cuestión puede ser resumida en la siguiente pregunta: jes de interés nacional incentivar la creación de un sistema procesal armónico para la resolución de las controversias transnacionales?

No debe ser descartado fácilmente o por temor, no solamente por el imperialismo norteamericano, sino también por las potencias financieras y comerciales de Europa. Esta cuestión no es tan simple cuando no parece otorgar respuestas fáciles. Un instrumento que facilite la circulación jurídica, necesariamente facilita y consolida la circulación comercial y, 
consecuentemente, el mantenimiento y potenciación del esquema comercial basado en la exploración política y económica de los países menos desarrollados ${ }^{21}$.

Por otro lado, en la medida en que ese comercio libre y desenvuelto con las grandes potencias sea considerado favorable también para los países menos desarrollados, en la medida en que la libre circulación comercial atraiga inversiones y fortalezca las relaciones comerciales, la iniciativa de este proyecto es igualmente valiosa. Una actitud participativa y al mismo tiempo alerta por parte de los países menos desarrollados es, tal vez, la mejor postura que debe ser adoptada.

El autor tiene serias dudas sobre los cuatro problemas antes reseñados (y aún sobre otros). Se podría decir que los propios relatores del proyecto, Profs. Hazard y Taruffo, comparten por lo menos algunas de nuestra dudas, dado que, como vimos, se trata de un trabajo en pleno camino, pasible de ser reformado y hasta de su pleno abandono.

Se espera, sin embargo, que un riguroso análisis de los problemas antes mencionados estimulen más de una adecuada comprensión del fenómeno e incentive la producción de críticas bien intencionadas y más útiles al desarrollo del propio proyecto.

\section{Conclusión}

El proyecto busca, tal vez, aún una utópica o ingenua armonía entre el proceso civil de los países del Civil Law y los países del Comon Law, pero eso es lo que sus autores están desarrollando en su propio tiempo, y solo el tiempo lo podrá decir al final.

Se ha procurado maximizar los aspectos positivos de cada sistema, minimizando los aspectos negativos. Eso no siempre fue posible, sin embargo en base a la necesidad de realizar compromisos o con el objetivo de satisfacer a los sistemas de todos los países. La iniciativa de encontrar un «menor denominador común» entre los diversos sistemas no revelan

21 En el mismo sentido, a contrario sensu, ver Constantino Kerameus, "Procedural unification: the need and the limitations, en I.R. Scott (ed.) International perspectives on civil justice, p. 50, justificando el Proyecto de aproximación del proceso civil en la Comunidad Europea bajo el fundamento que la diversidad de normas procesales ocasiona distorsiones en la libre competencia y alteraciones en la circulación de las mercaderías. 
necesariamente una menor disciplina para un determinado instituto procesal, y puede al mismo tiempo generar una solución insatisfactoria para todos los sistemas involucrados.

La opción de los relatores fue, declaradamente, la de adoptar el régimen de la estructura procesal de los países del Common Law como punto de partida, con el cuidado de evitar los «excesos» del derecho procesal americano. ${ }^{22}$

Se adoptó, así, una estructura procesal inexistente en los países del Civil Law, en la que el proceso está dividido en dos fases: una fase de investigación de las pruebas (discovery), y una fase de audiencia (trial o plenary hearing). La primera fase esta conducida directamente por los abogados, sin participación directa del juez, ${ }^{23}$ con el objetivo de obtener las pruebas e informaciones que están en manos de la parte contrario o terceros. En la segunda fase, se presentarán esas pruebas en la audiencia para ser valoradas por el Juez de la causa.

En base a esta marcada preeminencia del sistema procesal del Common Law en las normas transnacionales, algunos de sus aspectos son incomprensibles y casi imposibles de traducir para el procesalista del Civil Law. La dificultad de traducción no debe ser subestimada y no solamente esta referida a la imposibilidad de hallar un vocabulario técnico adecuado para la traducción de un término o de un instituto. El real problema es que algunos de los institutos son simplemente desconocidos o diferentemente delineados en diferentes países o en diferentes sistemas. ${ }^{24}$

Además, el proyecto enfrenta algunos problemas procesales que son inexistentes o desconocidos en la mayoría de los sistemas del Civil Law, principalmente en los países de América Latina, como en el caso del Perú. En algunos casos, se legisla en el vacío, con normas que no son de decisiva aplicabilidad a los procesos instaurados en el sistema latinoamericano. En

22 Como sabemos, el derecho procesal civil americano es diferente de los demás sistemas procesales del Derecho Común derivados del sistema inglés, principalmente en lo que se refiere al juzgamiento por jurado en las causas civiles y en los procedimientos de Discovery (que podrían traducirse en el Civil Law como de "prueba anticipada" o "diligencias preparatorias"). Otras características importantes del mismo son también la selección de los magistrados (a través de: elección, afiliación partidaria, previa experiencia profesional), una mayor flexibilidad de las normas procesales y el reglamento de los honorarios de los abogados (autorización de pacto de la cuota litis y la inexistencia de la regla general del pago de las costas y costos procesales a cargo de quien resulte perdedor en un proceso).

23 El juez debe ser llamado a intervenir únicamente en caso de conflicto

24 Cf. Normas transnacionales, Nota 1 de traducción. 
otros casos, se legisla insuficientemente, creándose nuevos institutos, sin prever su disciplina completa.

En muchos aspectos, se crea un sistema más exótico para el litigante del Civil Law que de cualquier otro sistema existente en un país de tradición diferente al Civil Law. Por ejemplo, para el litigante italiano será, en ese sentido, más conveniente y menos estresante operar sobre las leyes procesales francesas, españolas o alemanas que sobre las normas transnacionales. Dígase lo mismo del litigante brasileño, uruguayo, peruano o argentino en cualquier otro país de América Latina. Eso mismo es de esperar que, en estos casos, a menos que quiera hacer uso de algún instrumento previsto en las normas e inexistente en la ley interna (como el discovery, por ejemplo), el litigante opte por la legislación interna.

Exactamente por ese motivo, y prerrogativa de la parte extranjera de optar entre la aplicación de la norma procesal interna o de las normas transnacionales. En efecto, el sistema de las normas transnacionales es concebido en función de las expectativas de la parte extranjera y de la parte nacional, y no podrá serle impuesto el sistema de las normas, recusando la legislación procesal de su propio país. ${ }^{25}$

Si bien es verdad que el proyecto esta muy lejos de ofrecer un adecuado equilibrio entre los diversos sistemas para establecer una disciplina adecuada que rija al proceso civil en un país del Civil Law, es imperativo reconocer que este tiene el innegable mérito de introducir técnicas, ideas e institutos desconocidos en ambos sistemas, principalmente en los del Civil Law. Por ese motivo, las normas transnacionales serían mucho más útiles como un "sistema procesal modelo", que de ser tratado como una ley procesal especial para los litigios internacionales.

Será sobre este ropaje que el proyecto prestará su mejor contribución para una eventual armonización de las normas procesales en este milenio que ya se inicia. En la medida en que estemos, todos nosotros, dispuestos a aprender nuevas técnicas y a introducir y aplicarlas en nuestro sistemas, mejoraremos nuestro derecho en una misma dirección y nos estaremos aproximando, a ser, todos nosotros, cada vez menos, vecinos distantes. 